\title{
Modeling the Dynamics of MEMS-based Mobile Devices under Speed-controlled Human Motion Using Acceleration Measurements
}

\author{
Andreas Kotsopoulos and Theodore Antonakopoulos \\ Department of Electrical and Computers Engineering \\ University of Patras, Rio-Patras, 26500, Greece \\ Email: akotsop@ece.upatras.gr, antonako@upatras.gr
}

\begin{abstract}
This work focuses on modeling the dynamics of MEMS-based mobile devices, when are subject to speedcontrolled human motion, using measurements from accelerometers mounted on the device's frame. The measurement procedure and used data acquisition setup, indicative measurements showing the effect of physical system characteristics on the resulting acceleration signal diversity, the collected data analysis, as well as the selected modeling approach based on Hidden Markov Models (HMM) are presented and analyzed. Finally, simulation results of the proposed model's performance are discussed.
\end{abstract}

Index Terms-MEMS, HMM, Modeling, Dynamics, Acceleration.

\section{INTRODUCTION}

The constantly shrinking size of mobile devices together with the new requirements of emerging consumer applications has led to an apparent need for new, more compact and higher density storage technologies. Micro-Electro-Mechanical Systems (MEMS)-based storage technology appears to be a promising approach offering attractive features such as extremely high densities, low power consumption and low cost. The major MEMS storage projects at IBM Research [1], HP Laboratories [2] and Carnegie Mellon University [3] use different recording technologies, but similar mechanics, comprising of arrays with hundreds or thousands of probes positioned above the storage medium. The arrays are activated simultaneously and move short precise distances laterally and longitudinally using an electromechanical nanopositioning system of sensors and actuators.

However, the inherent mobility of the data recording mechanism makes these systems prone to oscillations and positioning inaccuracies caused by external acceleration disturbances imposed on most mobile devices. Furthermore, considering the nanometer-scale precision limits for reliable information recording/retrieval [4], significant burst-errors are highly likely to occur leading to overall system efficiency degradation. Due to their vast variety in form and time-scale, such disturbances cannot always be adequately grouped and modeled. In some cases, however, such as human motion acceleration, which can be considered as a rather frequent disturbance source owing to mobility, a coarse pattern periodicity can be identified. Thus, a probabilistic signal generation model can be extracted, capable of producing human motion acceleration patterns and variants

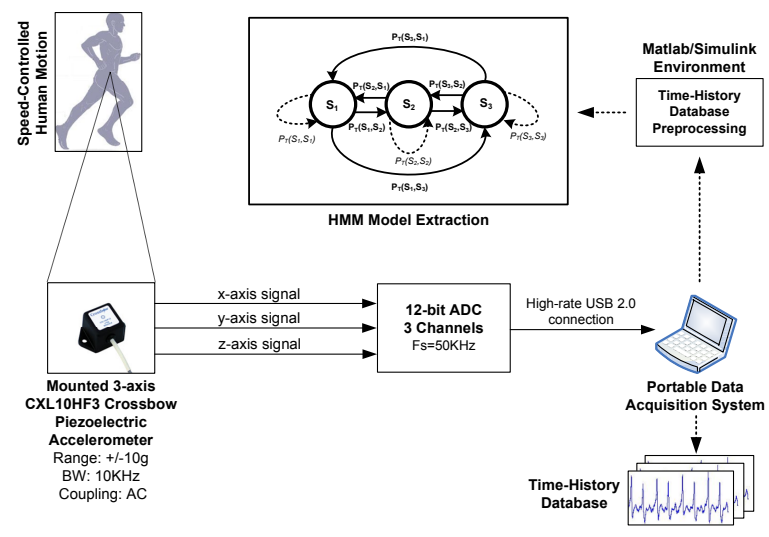

Fig. 1. Human running/walking motion acceleration experimental setup and data acquisition system.

while preserving basic statistical characteristics and shapeprofiles close to those of measured actual signals.

The necessity of such a model is twofold: first it provides a tool for generating various acceleration time-series profiles in the laboratory environment as input to both system-modeling in simulation and device-testing on vibration testing equipment such as vibration tables. Second, in typical MEMS-based storage systems, information is organized in sectors, with each sector recording/retrieval operation time-span being in the order of some microseconds [1], which is clearly outgrown from the human motion pattern's period (order of hundrends of msecs) by at least one order of magnitude as will be shown in Section III. Based on this, it can be readily assumed that during a single access operation, the device is inside a specific region of the pattern. Thus, the a priori knowledge of the disturbance characteristics can be exploited in order to improve several subsystems. In positioning control algorithms, for evaluation, prediction of highly probable forthcoming disturbance conditions, and appropriate parameter-adaptation, while in errorcorrection schemes for determining the profiles/statistics of burst-errors and algorithm evaluation. Related work on the area of vibration modeling includes a variety of approaches [5], [6], [7], [8]. However, they mainly address this topic at different dynamic ranges, modeling targets and time-scales.

The main purpose of this work is the measurement, analy- 


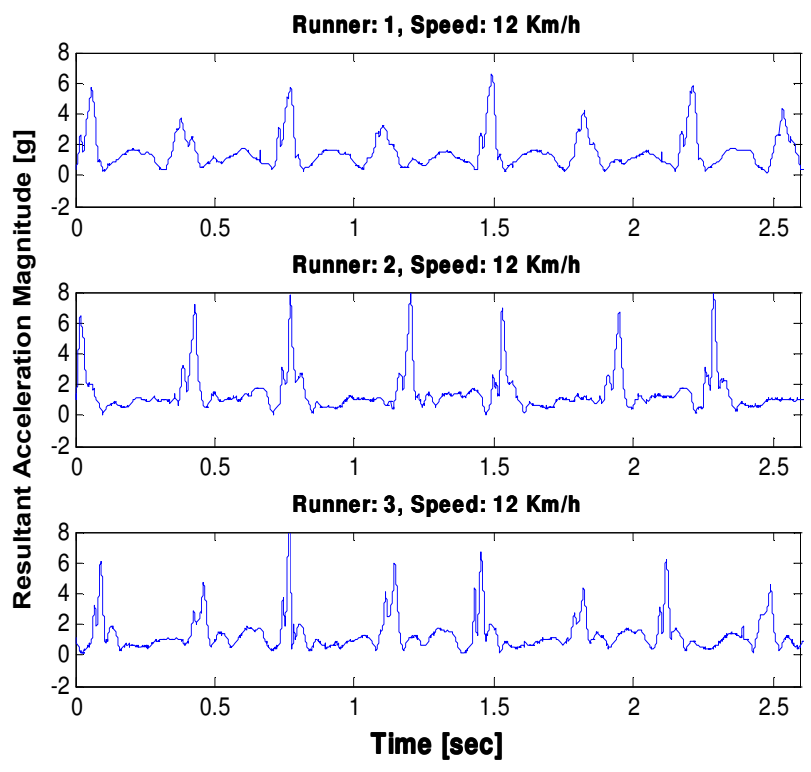

Fig. 2. Resultant acceleration magnitude measurements for three different runners at $12 \mathrm{Km} / \mathrm{h}$.

sis and modeling of human motion acceleration (specifically walking or running) imposed on mobile devices. This is achieved by mounting the accelerometer directly on the frame of the mobile device, aiming at a versatile statistical model able to describe the inherent diversity of such disturbance environments and to predict the short-term acceleration conditions in an operating device. In Section II, the experimental setup consisting of the measurement and data acquisition equipment used is presented and the carried out measurement scenarios are outlined. In Section III extensive measurements from several scenarios, the collected dataset analysis and preprocessing steps are presented. Section IV, describes in detail the modeling approach and the resulting statistical model, while simulation results showing the proposed model's performance are shown in Section V. Finally, in Section VI we present future goals on improving and extending the model.

\section{Experimental Setup And Measurements}

An extensive acceleration time-histories ${ }^{1}$ dataset was obtained using the experimental setup shown in Figure 1. The measurement equipment used was a 3-axis high-bandwidth accelerometer mounted on a typical commercial mobile device in order to avoid undesired measurement-noise caused by friction and vibrations between the device and the accelerometer. The measurement system was placed on a running person's waist using a commercial mounting accessory provided by mobile device manufacturers, in order to measure the acceleration imposed on the device under real conditions. Data acquisition was performed using three simultaneously accessed, highbandwidth 12-bits resolution Analog-to-Digital Conversion (ADC) channels sampled at $50 \mathrm{KHz}$, providing a sufficient

\footnotetext{
${ }^{1}$ Acceleration waveforms over time
}

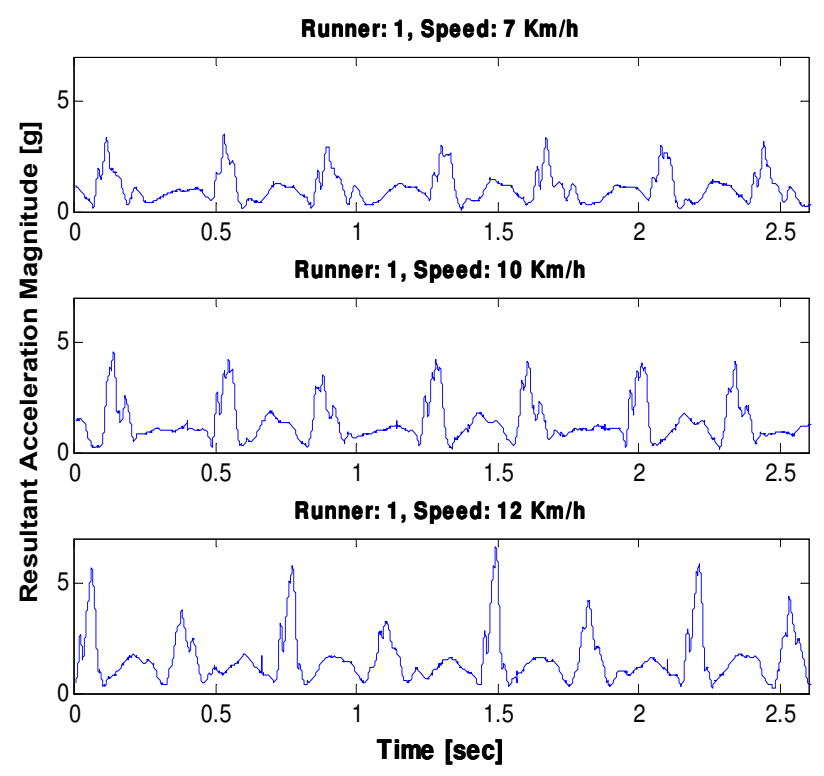

Fig. 3. Resultant acceleration magnitude measurements for Runner 1 at 7 , 10 , and $12 \mathrm{Km} / \mathrm{h}$.
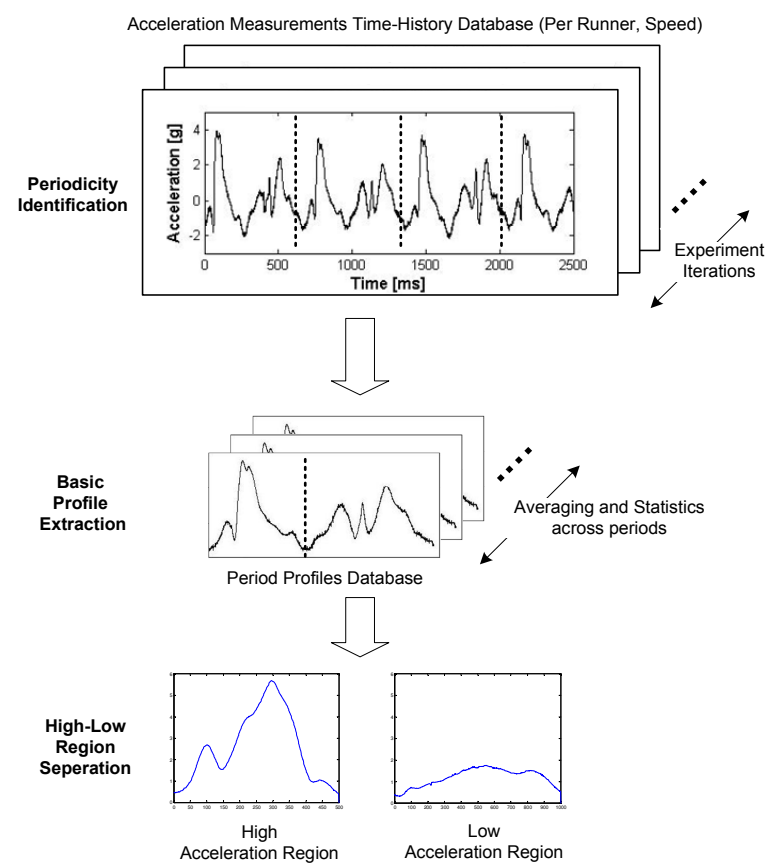

Fig. 4. Acceleration measurement data analysis. The database is reformed containing High and Low acceleration region profiles.

oversampling rate of 5 with respect to the accelerometer's bandwidth.

Acceleration data were collected from 7 different runners (Runner 1 - Runner 7) with different physical characteristics, walking/running at 8 controlled speeds $(5 \mathrm{Km} / \mathrm{h}-12 \mathrm{Km} / \mathrm{h})$ in a commercial treadmill. Four 2.6 secs-long time-histories per runner per speed were acquired. This way a large measurement dataset was created, using the resultant acceleration vector magnitude from all three axis, since the overall acceleration profile exerted on the device is that of interest. Measurement 


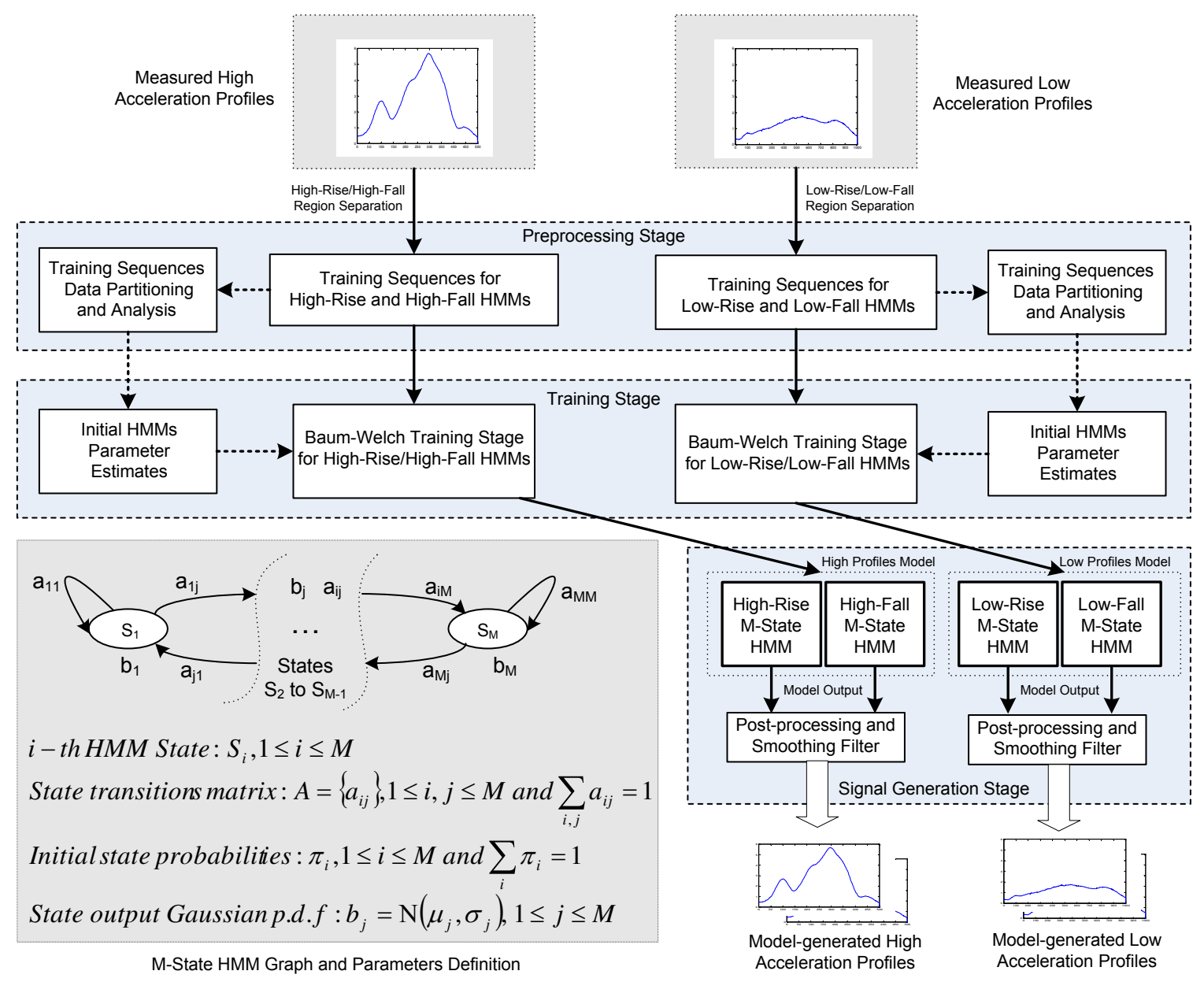

Fig. 5. HMM-based statistical modeling and signal generation procedure.

results are presented in Figures 2, 3, showing the change in the signal's characteristics under several scenarios, such as running speed, running style and runners' physical characteristics. From these figures, the inherent periodicity of the physical phenomenon is also obvious, which confirms the second necessity described in I.

\section{Measurement Analysis and Preprocessing}

The statistical analysis of the measured signals has lead to the following observations regarding the physical processes' basic properties. Firstly, the acceleration signal progresses almost periodically in time through a recurrent coarse pattern, translating to the time-span between two successive footground contacts of the runner. Inside each pattern, one high and one low acceleration region can be identified linked together by a transition region. Secondly, the pattern's period dependence on either speed or runner's physical characteristics (among other limb length, fat body distribution, muscle size and strength, running surface hardness) is rather small and can be considered as negligible. On the contrary, the high, low, and transition regions change significantly with respect to both of the above mentioned factors, whereas their basic profile changes vastly between different runners but retains a basic shape with respect to speed changes for the same runner.

Based on the above observations, the acceleration signal's diversity makes it very difficult to extract one general model capable of describing all possible cases. The focus of this paper with respect to modeling resides on providing a methodology for extracting one statistical model per candidate runner from measurements, capable of describing sufficiently many basic shape variants of the high, which in fact is the one of major significance, and low acceleration profiles, while it can be later extended and generalized in order to include transition regions and coarse physical characteristics parametrization capability. Figure 4 depicts the decomposition of the analysis and preprocessing procedure applied on the acquired dataset for a specific runner, in order to construct an ensemble of high and low acceleration profiles, which will be used as training sequences for the model.

\section{HMM-Based Statistical Modeling ANd Signal GENERATION PROCEDURE}

The proposed Hidden Markov Model (HMM) [9] based modeling scheme, presented in detail in Figure 5, can be decomposed in three distinct stages: preprocessing, training and signal generation stage. Since the steps applied for high 
TABLE I

Comparative Statistics of MEAsured VERSUS Simulation Generated ACCELERATION Profiles For HMM-BASEd Models With 3, 5, 7 AND 9 STATES.

\begin{tabular}{|c|c|c|c|c|}
\hline \hline Model Comparison & mean & var & skewness & kurtosis \\
\hline Actual Measured Acc. Profile & 4.24 & 1.62 & -0.54 & 2.00 \\
\hline 3-State HMM Acc. Output & 4.16 & 1.26 & -0.40 & 2.21 \\
\hline 5-State HMM Acc. Output & 4.17 & 1.28 & -0.50 & 2.13 \\
\hline 7-State HMM Acc. Output & 4.26 & 1.46 & -0.51 & 1.90 \\
\hline 9-State HMM Acc. Output & 4.23 & 1.45 & -0.56 & 2.00 \\
\hline \hline
\end{tabular}

and low acceleration profile modeling are identical, analyzing these steps for one of the two cases suffices for a comprehensive description of the followed modeling procedure. For this reason, only the high acceleration profile modeling path will be discussed here, noting that the same procedure applies also for the low profile case.

In the preprocessing step, each high profile of the ensemble created from the measurements analysis presented in Section III is further subdivided into a rise (High-Rise) and a fall (High-Fall) region on the basis of the maximum reached acceleration magnitude point, with the resulting two ensembles serving as the training sequences for the training stage following. Additionally, a data partitioning and statistical analysis step is applied on each of the training sequence ensembles. In this step, the signal magnitude range is partitioned based on the number of HMM states used and the occurrence of acceleration magnitude fluctuations is statistically investigated. This procedure ends in an initial coarse estimate of the HMM parameters defined in the down-left side of Figure 5 , leading to improved convergence stability of the HMM training algorithm to a desired local maximum point. The training algorithm used was the Baum-Welch, which is an iterative two-step optimization algorithm [9]. Using the initial parameter estimates, the algorithm iteratively re-adjusts them and finally produces the maximum likelihood estimates for the parameters of each of the High-Rise and High-Fall HMMs given the observations training sequence ensembles. In the signal generation stage, the constructed High-Rise and High-Fall HMMs are used to generate the respective rise and fall regions of the high acceleration profile. The output waveforms from the two HMMs are combined and supplied to an appropriate smoothing filter in order to minimize large high frequency fluctuations produced by the Gaussian state output probability density functions. This way a single model-generated high acceleration profile is created.

The quality of the model-produced profiles depends highly on the number of states assigned to each of the two HMMs used. In order for a sufficient number of states to be determined, the above described modeling scheme was applied for the cases of 3, 5, 7 and 9 states in both High-Rise and High-Fall HMMs. The generated output efficiency with respect to the measured profiles was evaluated in terms of

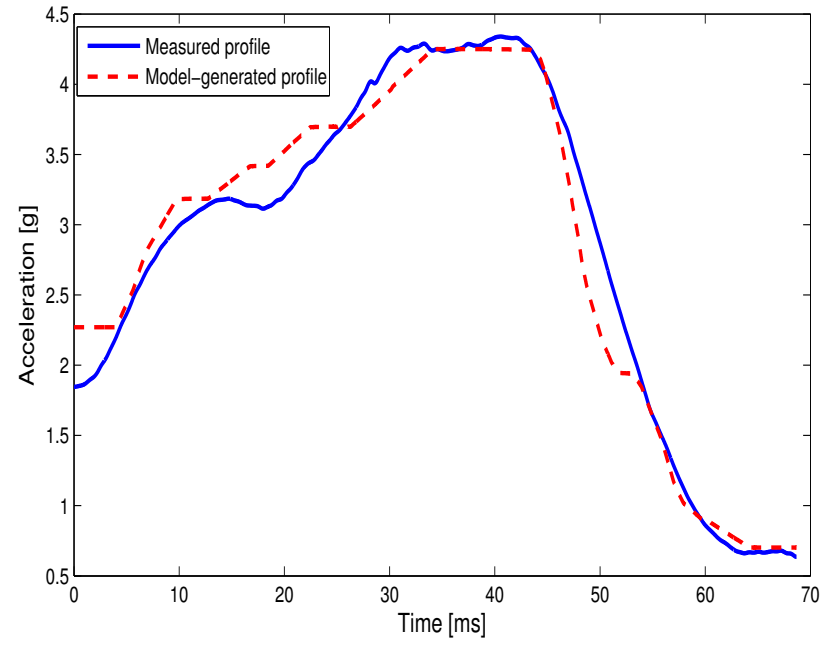

Fig. 6. Measured versus model-generated high-acceleration profile for the case of $10 \mathrm{~km} / \mathrm{h}$.

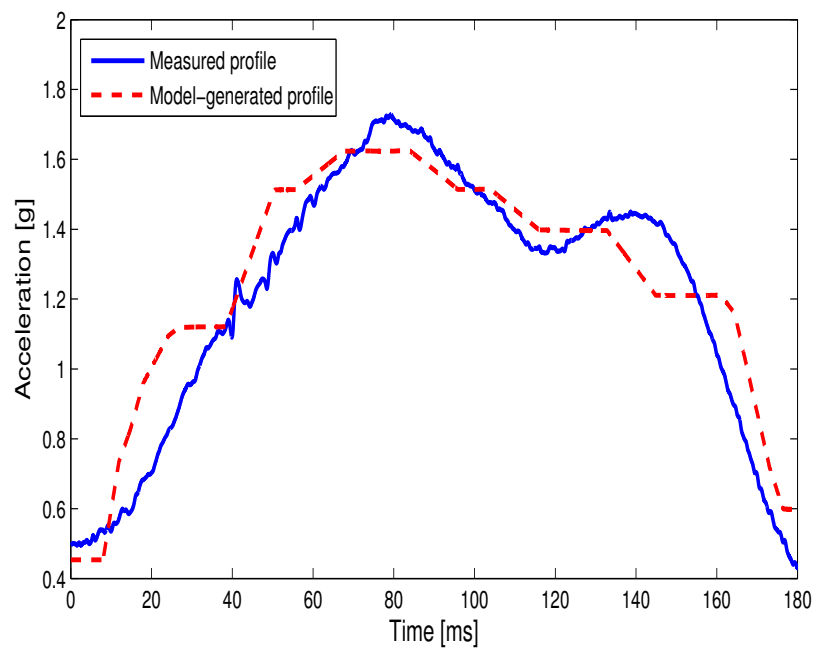

Fig. 7. Measured versus model-generated low-acceleration profile for the case of $10 \mathrm{~km} / \mathrm{h}$.

the first four statistical moments of their p.d.f for all four cases. Based on the comparative results listed in Table I and considering the trade-off between model complexity and model signal generation accuracy, the 7-state HMM case was chosen, since smaller models lead to significant statistical accuracy degradation and poor signal fluctuations description, whereas larger models lead to increased complexity without a corresponding improvement in the two previously mentioned factors.

\section{Simulation Results}

As previously stated, only the general profiles of low/high acceleration regions are retained, while several variants of them appear in different time-snapshots of the signal. This inherent stochastic nature of both the physical process to be modeled and of the constructed model itself implies that a direct one-to-one comparison between a specific low/high 


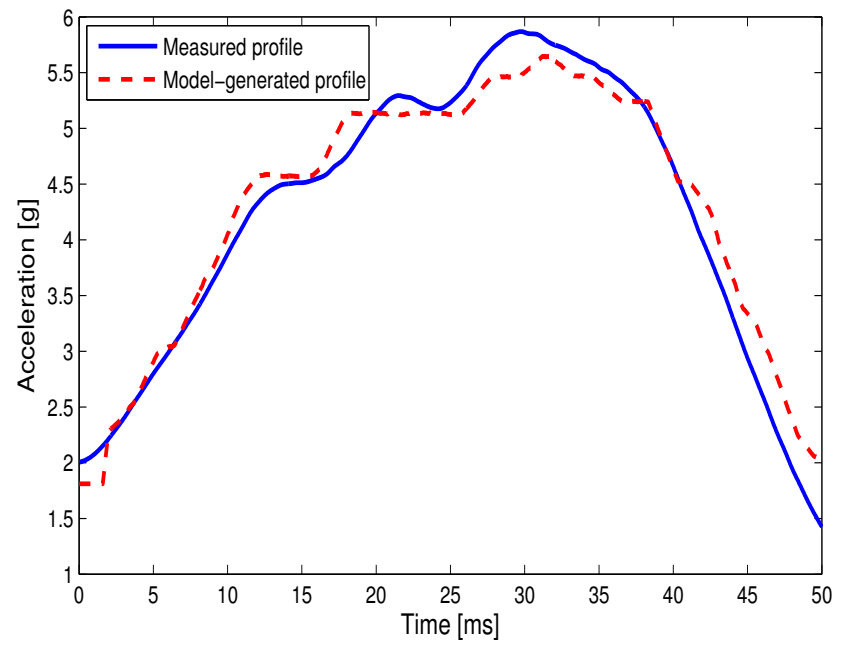

Fig. 8. Measured versus model-generated high-acceleration profile for the case of $12 \mathrm{~km} / \mathrm{h}$.

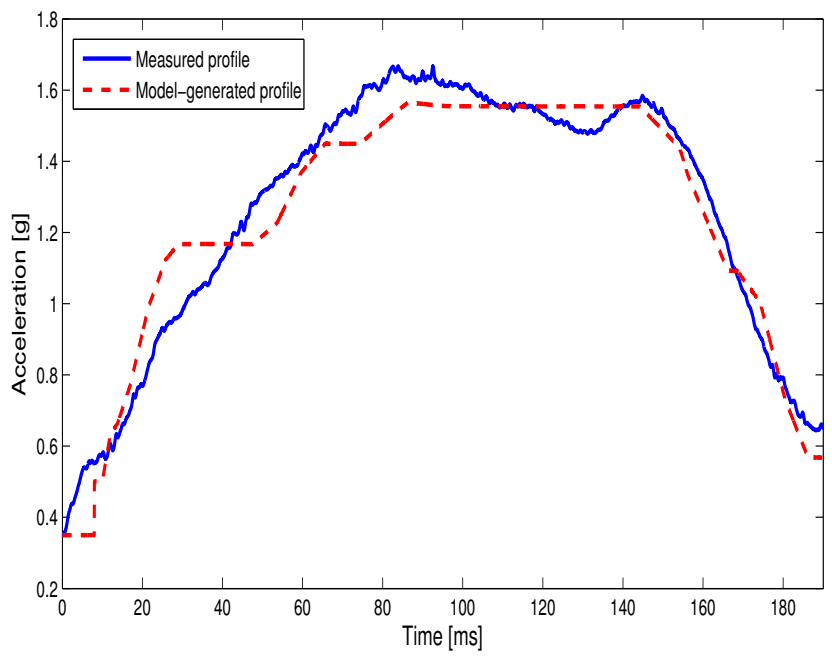

Fig. 9. Measured versus model-generated low-acceleration profile for the case of $12 \mathrm{~km} / \mathrm{h}$.

profile manifestation from measurements and a single modeloutput profile is not practical for model efficiency evaluation purposes. Instead, the mean low/high acceleration profiles generated by several iterations of the model are compared with the respective mean measurement-extracted profiles.

In that sense, the proposed modeling procedure analyzed in Section IV is applied for the randomly chosen case of Runner2 and its low/high acceleration profile generation capability is presented for two indicative speed values. Specifically, Figures 6 and 7 show mean high and low model-generated profiles versus actual measured mean profiles for the speed of $10 \mathrm{~km} / \mathrm{h}$. Comparative results for the case of $12 \mathrm{~km} / \mathrm{h}$ are presented in Figures 8 and 9 for high and low profiles respectively. The results show that in all cases the model's mean output profile is very close to the actual measured mean profile. Furthermore, the selected number of states for the underlying HMMs proves adequate in order for the model to capture the variations in the signal's magnitude inside each individual region. As a consequence, the proposed model is capable of producing a large number of general-shape-preserving acceleration profile variants, which in addition statistically sum up to a sufficiently accurate, with respect to the measured, mean profile.

\section{CONCLUSIONS AND OPEN ISSUES}

In this paper, the problem of creating a framework for modeling the acceleration imposed on mobile MEMS-based devices from human motion was addressed. Extensive measurements and comparative graphs showing the overall human motion acceleration dependencies, as well as the HMM-based modeling and signal generation approach were presented in detail. Finally, model-generated versus measured acceleration waveform profiles over time showing the proposed statistical models signal reconstruction capability, were presented and analyzed. Future goals on the subject concentrate on extending the current model so as to describe signal low-to-high transition regions as well as basic runner cases through appropriate model-parameter adjustments.

\section{REFERENCES}

[1] A. Pantazi, A.Sebastian, T. Antonakopoulos, P. Bächtold, A. R. Bonaccio, J. Bonan, G. Cherubini, M. Despont, R. A. DiPietro, U. Drechsler, U. Dürig, B. Gotsmann, W. Häberle, C. Hagleitner, J. L. Hedrick, D. Jubin, A. Knoll, M. Lantz, J. Pentarakis, H. Posidis, R. C. Pratt, H. Rothuizen, R. Stutz, M. Varsamou, D. Wiesmann, and E. Eleftheriou, "Probe-based ultrahigh-density storage technology," IBM J. Res. and Dev., vol. 52, no. 4/5, pp. 493-511, 2008.

[2] M. U. Arif, M. Guillermo, and A. Alvarez, "Using MEMS-based storage in disk arrays," in 2nd USENIX Conference on File and Storage Technologies, San Fransisco, CA, Mar. 2003.

[3] Carnegie mellon university project database. [Online]. Available: http://www.dssc.ece.cmu.edu/research/projects/40

[4] H. Pozidis, W. Häberle, D. Wiesmann, U. Drechsler, M. Despont, T. Albrecht, and E. Eleftheriou, "Demonstration of thermomechanical recording at 641 Gbit/in ${ }^{2}$," IEEE Transactions on Magnetics, vol. 40, no. 4, pp. 2531-2536, Jul. 2004.

[5] T. Obata and Y. Miyamori, "Identification of a human walking force model based on dynamic monitoring data from pedestrian bridges," Computers and Structures, vol. 84, pp. 541-548, 2006.

[6] R. Bowden, "Learning Statistical Models of Human Motion," in IEEE Workshop on Human Modeling, Analysis, and Synthesis, CVPR, 2000.

[7] B. Das and Sengupta, "Computer-aided human modelling programs for workstation design," Ergonomics, vol. 38, pp. 1958-72, 1995.

[8] D. Tolani, A. Goswami, and N. Badler, "Real-time inverse kinematics techniques for anthropomorphic limbs," Graphical Models, vol. 62, no. 6, pp. 353-88, 2000.

[9] L. R. Rabiner, "A Tutorial on Hidden Markov Models and Selected Applications in Speech Recognition,” vol. 77, no. 2, February 1989. 